\title{
XIV.
}

\section{ZUM ANDENKEN}

\section{an \\ WILHELM BAUM.}

\author{
Ein Nekrolog
}

von

Professor Dr. Edmund Rose in Berlin.

Am 6. September dieses Jahres hat die deutsche Chirurgie ihren Senior, unsere Zeitschrift einen ihrer Herausgeber, die Chirurgie einen ihrer stillen, aber darum nicht weniger wirksamen Pfleger in Deutschland verloren. Es sei mir erlaubt, Wilhelm Baum hier einige Worte des Andenkens zu widmen, um so mehr, da er, ein Mann von der Art eines Schönlein und Wilms, weniger durch die Schrift sich selbst ein Denkmal gesetzt hat, als durch seine Persönlichkeit von Einfluss auf die Entwicklung unserer Kunst und Wissenschaft gewesen ist. Baum ist beinahe 84 Jahre geworden und hat trotzdem bis fast zuletzt gewirkt.

Aeltere Kreise muss man reden hören, wenn wir von seiner praktischen Wirksamkeit erfahren wollen; war er doch, ein Dutzfreund von Dieffenbach und Karl Mayer, der Letzte dieser Generation. Dann später in Göttingen verbreitete sich sein Ruf als Lehrer zu einer Zeit, wo in Deutschland wenige Vorträge der Chirurgie Anziehungskraft hatten; beneidet wurde unter uns der, dem sein Geschick erlaubte, ihn aufzusuchen. Bis zu seinem Ende aber blieb er der theilnehmende Freund so vieler jüngerer Forscher, die er durch seine Kritik spornte, durch seinen Widerspruch reizte, gelegentlich auch tröstete, dann wieder durch seine seltene Belesenheit beschämte, jedenfalls aber dadurch erfreute, dass man sah - was doch heutzutage selten geschieht - immer hatte er die Sachen wirklich gelesen, über die er urtheilte.

So konnte es nicht fehlen, dass, wenn $B$ a u m auch auf dem Berliner Chirurgencongress sich wenig zur (xeltung brachte und selten anwesend 
war, doch, sobald er kam, sein anregender Umgang gerade für so manchen juingeren Forscher, weit uiber den Kreis seiner Schüler hinaus, der wesentliche Anziehungspunkt dieser Vereinigung war, so oft er fehlte, stets schwer vermisst wurde.

Charakteristisch ist es vielleicht für Baum, wie Schreiber dieser Zeilen das Glïck hatte, mit ihm bekannt zu werden. Es war in meiner Assistentenzeit in Bethanien (1863), dass Wilms einmal einen älteren Herrn aus der Stadt mitbrachte und die ganze Visite in ungewohnter Genauigkeit mit ihm machte; als $\mathrm{W}$ il ms sich verabschieden wollte, winschte er doch noch auch zu mir geführt zu werden, um meinen Farbenmesser zu sehen. Ueber zwei Stunden hatte ich ihn schon durchs Haus begleitet, unbekannterweise, da es früher in Bethanien nicht üblich war, die Assistenten vorzustellen. Jetzt kam er noch besonders auf mein Zimmer, liess sich auf das Genaueste den Apparat zeigen und gewiss über eine Stunde zu dem, was er schon davon gelesen, auch noch das auseinandersetzen, was von meinen Arbeiten iiber Farbenkrankheiten gerade im Druck war. Zuletzt musste ich ihm noch ein Exemplar für die Göttinger Klinik anfertigen lassen. Wie glücklich mich dieser Besuch in meinem obscuren Dasein in Bethanien damals gemacht hat, brauche ich kaum zu sagen; ausser Gräfe und meinem unbekannten englischen Uebersetzer in dem Philosophical Magazine ist Ba um doch wohl der Einzige gewesen, der diese Arbeiten wirklich gelesen hat. Aehnliche Theilnahme hat, wie ich weiss, Mancher unter uns Jüngeren bei ihm gefunden; kann man sich wundern, dass seine scharfe, aber doch so liebenswürdige und anregende Kritik ihm unser Herz gewann?

Dass Baum aber auch schon vor unserer Zeit mit der Generation, aus deren Schriften wir alle unsere Bildung genossen, in gleich offenem und mittheilsamem Verkehr anregend und fördernd auf die Wissenschaften gewirkt hat, wen könnte man da besser als Zeugen anführen, als unseren Wilhelm Roser. Erinnern wir uns, was er schon 1859 in der Vorrede zur dritten Auflage seines Handbuches der anatomischen Chirurgie sagt:

„Es geschieht jetzt häufig und wohl häufiger als je, dass ein Schriftsteller die Ideen oder Erfahrungen, welche er von Anderen mitgetheilt erhalten hat, als die seinigen hinstellt. Vor diesem Vergehen habe ich mich sorgfiltig zu hüten gesucht; da aber dasselbe auch unbewusst vorkommen kann, so bin ich nicht sicher, ob es mir ïberhaupt gelungen ist, jeden Vorwurf in solcher Beziehung ganz zu vermeiden. Bei der grossen Erleichterung des Verkehrs, welche jetzt stattfindet, kommt es öfters vor, dass durch mündliche Mittheilungen und durch persönlichen Ideenaustausch Gedanken angeregt und durch Erfindungen gefördert werden, von denen man in der Folge nicht mehr sagen kann, wer eigentlich deren Urheber sei. Ueberhaupt lernen die Collegen viel von einander im 
persönlichen Verkehr. Den grossen Vortheil, sowie den geistigen Genuss, welche hieraus entspringen, habe ich immer sehr hoch angeschlagen und viele Förderung, Anregung und Belehrung dabei gewonnen. Ich bin auch vielen Collegen dafür zu lebhaftem Dank verpflichtet. Ich glaube aber eine besondere Quelle der persönlichen Belehrung und Förderung, welche mir offenstand, besonders nennen zu müssen. Es ist dies der Verkehr mit dem hochverehrten Fachcollegen in Göttingen, Herrn Hofrath W. Baum. Es war mir gestattet, aus der umfassenden Erfahrung und der vielseitigen Literaturkenntniss, welche dieser hochgeschätzte Freund besitzt, viel nuitzliche und werthvolle Belehrung $z \mathfrak{u}$ ziehen und ich bin dadurch zu einer grösseren Sicherheit im Urtheil und zur Berichtigung mancher einseitigen Ansicht gelangt. Ieh folge nur dem Gebot der Dankbarkeitspflicht, indem ich dies hier öffentlich bekenne und ausspreche."

Das sind aus früherer Zeit Worte der Anerkennung, welche von seinem nächsten klinischen Nachbar in Marburg Baum zu Theil wurden.

Möge es mir gestattet sein, was mir über Baum's Anfänge und seinen äusseren Lebensgang bekannt geworden ist, bei dieser Gelegenheit noch kurz anzufïhren.

Geboren am 10. November 1799 in Elbing, verlor er seinen Vater, Samuel Gottlieb Baum, welcher dort Kaufmann und Stadtrath war, schon friih, im Januar des Jahres 1822, wenige Monate vor seiner Promotion. Liest man, dass seine Mutter, eine geborene Cabrit, zur französischen Colonie gehörte, so wird man dabei an die ihm eigene Lebendigkeit und Regsamkeit im Umgang erinnert. Nachdem er von 1809-1818 das Gymnasium in Elbing besucht, studirte er ein Jahr an der heimischen Universität, in Königsberg in Preussen, wobei er zugleich sein Jahr abdiente und Mitglied des plilologischen Seminars unter Lobeck war. Osteologie hörte er damals bei dem späteren russischen Staatsrath v. Bär, mit dem er bis zum 'Tode in engster Freundschaft blieb, daneben unter Anderen bei $\mathrm{Herbart}$ verschiedene philosophische Vorlesungen, bei Bessel Integralrechnung, bei $\mathrm{Hag}$ en Experimentalphysik, bei Burd a c b Anatomie. Ende 1819 ging er nach Göttingen, wo er Himly, Langenbeck, Osiander, Blumenbach und Stromeyer hörte und Anfang 1822 nach Berlin, um Link, Berends, Rust, Gräfe und Siebold kennen zu lernen. In Göttingen hatte er einen lehrreichen Fall von Epispadie gesehen und auf Himly's Aufforderung Abbildungen davon gemacht. Rudolphi verschaffte ihm hier in Berlin einen zweiten Fall der Art und stellte ihm seine so reichhaltige Fachbibliothek zur Verfiigung, wie sie jetzt aus seinem Nachlass einen Schatz der königlichen Bibliothek in Berlin bildet. So entstand dann die berühmte ${ }^{1}$ ) Dis-

1) Vgl. darüber die Recensionen in Allg. medic. Annalen. 1824. S. 406; und Bibliothek d. prakt. Heilkunde v. Hufeland und Osann. 1823. Bd.50. S. 181. 
sertation von Baum: „De urethrae virilis fissuris congenitis speciatim vero de epispadia " (c. 3. tab. Berolini. 1822. Formis Brueschckianis 4), die heutzutage so schwer im Buchhandel zu haben ist. Sie enthält die Geschichte der Epispadie nach genauem Studium der zwei neuen, von ihm beschricbenen und der sonst in der Literatur aufzufindenden Fälle, ist mit drei Stahlstichen nach Zeichnungen von B a u m's Hand erläutert und bildet ein Glied jener schönen Reihe von werthvollen Dissertationen über die angeborenen Krankheiten, welche damals unter der Aegide von $\mathrm{Ru}$ dolphi in Berlin ersehien und der jungen Universität zur Zierde gereichte. Es waren das die Vorarbeiten zu einem grossen Werk tiber Missbildungen, zu welchem $R u d o l p h i$ selbst dann aber leider nicht mehr gekommen ist, während uns zum Glück wenigstens der ganze, von ihm gesammelte Schatz einsehlägiger Schriften erhalten ist.

Im Winter 1822 wohnte B aum in der Gräfe'schen Klinik, wie es scheint, als freiwilliger Assistent, machte 1823 nach dem Tode seiner Mutter sein Staatsexamen und ging dann auf Reisen, zuerst nach Wien, dann auf ein halbes Jahr nach Italien, von da nach Paris, schliesslich blieb er ein Jahr in Grossbritannien. Nach dreijähriger Abwesenheit kehrte er nach Berlin zurück, half dort gelegentlich seinem Freunde Dieffenbach ${ }^{1}$ ) bei besonders wichtigen und neuen Operationen und bekam 1829 die Berufung zum alleinigen Oberarzt des Danziger Stadtlazareths; da er sich (1829) mit Marie Günther, Tochter des Oberbaudirectors Guinther, verlobt hatte, nahm er die Stellung an. Ein Jahr darauf (den 29. August 1830) heirathete er. Damals brach in Danzig die Cholera aus; in zwei kurz auf einander folgenden Epidemien kamen von aller Herren Ländern Commissionen dahin, um diese neue Krankheit kennen zu lernen; zugleich entwickelte sich ein sehr reger Verkehr mit den Behörden, so dass $\mathrm{Baum}$ ausserordentlich bekannt wurde.

Nach theoretischen Erwägungen hatte ein Physikus und Hofarzt im Königreich Hannover, Dr. Schwarz, Sauerstoffeinathmungen gegen die Cholera empfohlen; B a u m constatirte ihre Unwirksamkeit ${ }^{2}$ ). Den Hauptwerth in der Therapie legte er schliesslich auf die Behandlung der prämonitorischen Diarrhoe, auf die er besonders die Aufmerksamkeit lenkte. ${ }^{3}$ )

Später fand Baum in Danzig die Flimmerbewegung beim lebenden Menschen und zeigte sie an eben extrahirten Nasenpolypen dem Herrn Dr. Carl Theodor v. Siebold in Danzig, der ihre gemeinsamen Ver-

1) Vgl. Dieffenbach's Angaben in seinen Aufsătzen in Rust's Magazin für die gesammte Heilkunde. 1829. S. 106 und 1830. S. 282.

2) Seine Mittheilung darüber befindet sich in einem Brief an $\mathrm{Hufeland}$ in Hufeland's und Osann's Journal der praktischen Heilkunde. Berlin. Juli $183 \hat{1}$.

3) Vgl. in No. 12 von Hohnbaum und Jahn's medicinischem Conversationsblatt. II. Jahrgang 1831. 
suche uiber Flimmerbewegung 1836 in der medicinischen Zeitung des Vereins fiur Heilkunde in Preussen veröffentlicht hat. ${ }^{1}$ ) Baum verschaffte dazu Gelegenheit und Mittel an frisch amputirten Beinen und an Leichen.

Nachdem Baum einen Ruf nach Erlangen ausgeschlagen, ging er im Herbst 1842 nach Greifswald als Professor der Chirurgie und Director der chirurg. Klinik, an Stelle von M a n d t, welcher als Leibarzt des Kaisers nach Petersburg berufen wurde; hier blieb er bis Ostern 1849, wo er durch Bardeleben ersetzt wurde. In dieser Zeit fasste König Friedrich Wilhelm IV. den Entschluss, „ein Institut zur Ausbildung von Krankenpflegerinnen " zu errichten und damit eine eigene Krankenanstalt zu verbinden. Diesem längst gefühlten Bedürfniss, die Zahl der Krankenhäuser zu vermehren, sollte die Stiftung des Central-Diakonissenhauses Bethanien auf dem Köpniker Felde in Berlin entsprechen, welches in den Jahren 1845 und 1846 erbaut wurde. Baum wurde damals veranlasst, einige Monate in Berlin zuzubringen, um beim Bau und der Einrichtung seinen Rath zu ertheilen. Man wünsehte auch, dass Baum die Stelle eines chirurgischen Oberarztes in Bethanien uibernehme, die Verhandlungen zerschlugen sich aber zuletzt, wie man sagt, wegen der Leichenfrage, und wurden dann Bartels als Oberarzt und Wald als Chirurg hier in Bethanien angestellt. Wie angesehen er auch bei den Aerzten war, geht daraus hervor, dass, als sein Freund Dieffenbach während der Klinik vom Schlage getroffen wurde und so unerwartet starb, eine Reihe der angesehensten Berliner Aerzte eine Petition an das Ministerium einreichte, mit dem Wunsche, Baum zu Dieffenbach's Nachfolger zu ernennen.

Gleichzeitig nach Kiel und Göttingen berufen, zog er jedoch das letztere vor und blieb dort bis zu seinem Tode, indem er der chirurgischen Klinik bis zum Herbst 1875 vorstand. Daneben hatte er, als Ruete 1855 nach Leipzig zog, anch die Augenklinik übernommen und las Augenheilkunde bis zum Jahre 1867, in welchem er dieselbe an Schweigger abtrat. Nachdem er 1875 sein Amt niedergelegt, beschäftigte er sich mit dem Plan, eine Geschichte der Chirurgie zu schreiben, kam aber über die Vorstudien nicht hinaus. Seit 1876 war er Mitglied der kaiserlichen Leopoldino-Karolinischen deutschen Akademie der Naturforscher. „Bis zuletzt in voller geistiger Frische und voll Interesse für Wissenschaft, Kunst und Natur, bewahrte er seine warme Theilnahme für seine Mitmenschen, frei von allen Gebrechen des Alters. Fr starb nach kurzer Krankheit, nachdem er eine beschränkte Lungenentzuindung glïcklich überstanden hatte, an Altersschwäche den 6. September 1883. Sein Sohn ist der bekannte, jetzige Chefarzt des städtischen Krankenhauses in Danzig.

1) "Ueber die Flimmerorgane des Menschen“. Von Dr. v. Siebold in Danzig. 1836 in No. 28. 
Wir können diesen Abriss nicht schliessen, ohne noch eine eigenthüliche Seite von Baum hervorzuheben, die so recht zeigt, wie der rege Sinn für die Wissenschaft ihn nie verlassen hat. Das sind die zahlreichen Studienreisen, die er noch als Professor gemacht hat. So ging er 1841 nach Paris, um bei Leroy d'Etiolles die Lithotripsie kennen zu lemen, 1845 nach Wien, um bei Rokitansky pathologische Anatomie zu hören und bei skoda einen Curs über Percussion und Auscultation zu nehmen, in den fünfziger Jahren zu Gräfe, um Augenspiegeln zu lernen. Ebenso hörte er noch in Göttingen Chemie bei Wöhler, Physiologie bei Wagner, Physik bei Weber, Optik bei Listing mit dem ihm eigenen Eifer und Fleiss.

Geschrieben hat B a u m ausser seiner besprochenen Dissertation wenig. Am bekanntesten ist seine Arbeit über den Weichselzopf. Auch dieser so exacte Bericht wäre uns vielleicht nicht erhalten, wenn er nicht im amtlichen Auftrage verfasst und so 1843 aus den Ministerialacten von E $\mathrm{ck}^{1}$ ) veröffentlicht wäre.

Zur Erforschung des Weichselzopfs wurden seit 1831 anfangs in einem Kreislazareth, dann aber seit Ende 1840 in dem Stadtkrankenhause zu Danzig Weichselzopfkranke auf Staatskosten aufgenommen. Nachdem Eck drei von Baum beigefugte Krankengeschichten im Auszug und anscheinend mit Zusätzen im Sinne der Tagesmeinung mitgetheilt, folgt abgetrennt Baum's Bericht, welcher durch vergleichende Messungen und zahlreiche Versuche am Haar bei verschiedenen Altersklassen sowohl bei Gesunden, als auch bei Plicösen nachweist, dass in seinen Fällen allerdings die Haare dieser $1 / 6$ dünner waren, als sonst im Durchschnitt; eins war aber auch dicker, weshalb er auf diesen Unterschied keinen grossen Werth legen will. Sonst bestätigte sich aber keine einzige Angabe, die über wesentliche Abweichungen in den Haaren Plicöser aufgestellt war, sondern im mikroskopischen Bau, sowohl am Schaft wie an der Wurzel, in den Proportionen, in der Dehnbarkeit, in der Tragkraft waren sie, für sich genommen, ganz normal; zufällige Beimengungen von Fremdkörpern (allerlei Thiere und ihre Ueberreste, Schinnen, Staub aller Art u. dergl.) in Menge und Fettzunahme bei der chemischen Untersuchung bildeten das Einzige, was am Zopf im Ganzen auffiel. Uebrigens wurden zur chemischen Untersuchung zwei Zöpfe verwendet, die ex Plicakranken abgeschnitten hat, obne daraus einen Nachtheil hervorgehen zu sehen, ein Verfabren, gegen welches der „Plicametastasen “ wegen ja noch jetzt, nach ïber 40 Jahren, von den

1) Er ist, wie es scheint nicht zu seinem Vortheil mit Einleitung und $\mathrm{Zu}$ sätzen zu den Krankengeschichten versehen, im 61. Theil des vom Verein für Heilkunde in Preussen fortgesetzten Rust'schen Magazins für die gesammte Heilkunde mitgetheilt. 
Kranken in jenen Gegenden oft so energisch protestirt wird, obgleich seitdem von Bärensprung und Hebra so oft und laut die Weichselzopffrage als eine einfache Frage des Kämmens proclamirt worden ist.

Hebra rühmt auch ferner, dass Baum in Danzig neben Stannius und Wiegmann der Erste war, der die Kenntniss der Krätzmilbe in Deutschland verbreitete (S. 427).

Sonst habe ich über Baum's literarische Arbeiten nichts gefunden.

Gelegentliche Vorträge und Discussionen auf Naturforscher-Versammlungen (z. B. 1861 in Speyer, 1865 in Hannover, 1878 über Atresia ani urethralis in München) scheinen ausser in den amtlichen Berichten meist nicht im Drucke wiedergegeben zu sein, und lässt sich deshalb nicht viel darüber ermitteln; man weiss ja, wie ungenau solche Referate oft sind. Dagegen sind Mittheilungen aus seinen Kliniken mehrfach verschiedenen Orts erschienen, z. B. in der Dissertation von Wilhelm Georg $\mathbf{B}$ aum, in den Mittheilungen von Georg $\mathbf{F}$ ischer, in den Aufsätzen von Scholz in Blankenburg, Prof. Krause, J. Rosenbach, Caspar Schröder, Stubenrauch und so vielen Anderen.

Gerade diese fortlaufenden stillen, kleinen Beiträge, in denen er indirect dureh seine Schïler die Entwicklung unserer Kunst und Wissenschaft so fleissig gefördert hat, machen es schwer, B a um's Wirksamkeit auf unsere Generation richtig zu schätzen. In der Eile ist es fast unmöglich, zumal für den, der erst später gekommen, seinen Einfluss vollkommen zu würdigen. Doch will ich Einiges herauszuheben versuchen. B a um scheint der Erste gewesen zu sein, der eine Herzwunde geheilt und sie erkannt hat; später war er im Stande, den anatomischen Nachweis der Narbe dazu zu liefern. Irre ich nicht, so hat Baum den Croupschnitt in Deutschland eingeführt; jedenfalls hat er ihn schon häufiger ausgeführt zu einer '/eit, wo ihn die ersten Chirurgen nur dem Namen nach kannten, und zwar bei einer Epidemie, die in Greifswald 1844 und 1845 herrschte. Wie auffallend, wenn wir noch 1848 in Dieffenbach's Testament, seiner operativen Chirurgie, diese Indication zum Kehlschnitt kaum 1) erwähnt finden! Später in Göttingen, lesen wir, hat Baum von 31 Kindern 12 (also 39 Proc.) damit das Leben gerettet, ein Verhältniss, das noch heute zu den besten rechnet. Ebenso hervorragende Resultate im Steinschnitt wurden damals von ihm berichtet (5 Heilungen

1) Bei Betrachtung der Indicationen sagt Dieffenbach (Bd. 2, S. 345): "Ausserdem hat man die Operation wohl angerathen und auch mitunter mit Glück ausgeführt bei krankhaften Bildungen in der Luftröhre, wie bei der Angina membranacea, bei der Laryngitis u. s. w. u. s. w. " Mit diesen Worten hat Dieffenbach noch 1848 die ganze Lehre vom Croupschnitt abgemacht, der heutzutage in Bethanien mehr als die Hälfte aller Operationen ausmacht; im Jahre 1882 wurden allein zweihundertundneunzig Croupschnitte in Bethanien vorgenommen. 
unter 6 Fällen). Eine der ersten Heilungen von Ileus durch die Operation der Enterotomie rührt von Baum her, der zweite Fall, in dem in Deutschland Jemand aus der Gefahr, am Kropf zu ersticken, befreit ist, gehört Banm an. Und so liesse sich gewiss noch vieles zusammentragen, woran man seine kräftige Mitarbeit an dem heutigen Zustand unserer Kunst und Wissenschaft ermessen könnte, wenn er auch nicht das laute Wort in der Literatur geführt hat. Aber auch das ist ja bald vergessen!

Unvergessen wird aber sicher Baum's Andenken Allen bleiben, denen er im Leben sein Wohlwollen und seine Theilnahme an ihrem Streben gezeigt hat, wie das in Bethanien nicht nur den Chirurgen, sondern schon der Anstalt zu Theil geworden. 